\title{
显式完全平方守恒差分格式的构造 及其初步检验*
}

\author{
王城**季仲贞 \\ (中国科学完大七物理研究所大乙数值模报开放实验室,北京 100080)
}

\section{关谯词完全平方守恒、完全能量守恒、显式差分格式}

构造完全平方守恒型差分格式是克服非线性计算不稳定的重要措施之一 和海洋问题的数值模拟中已得到广泛的应用 ${ }^{[4-61}$. 以往构造的完全平方守恒的差分格式一般 是隐式的, 而具体求解时总是化为显式方式近似地求解. 既然如此, 能否直接构造出显式的完 全平方守恒或完全能量守恒的差分格式呢? 答案是肯定的. 在文献 [1]的基础上,本文采用加 耗散的方式构造了多种形式的显式完全平方守恒的差分格式, 并用 Rossby-Haurwitz 波进行 了初步的检验,结果令人满意.

在本文中,考虑如下形式的算子微分方程:

以及与其相应的差分算子方程:

$$
\frac{\partial u}{\partial t}+\mathscr{L}_{u}=0
$$

$$
\frac{u^{n+1}-u^{n}}{\Delta z}+L u^{n}=0
$$

容易证明如下定理:

定理 1 若成立关系式

$$
\Delta x\left\|L u^{*}\right\|^{2}-2\left(L u^{*}, u^{*}\right)-0,
$$

则 (2) 式为显式平方守恒差分格式. 其中内积 $(F ; G)-\sum_{k} \rho_{k} F_{k} \cdot G_{k} \Delta \sigma_{k}, \rho_{k}$ 为权函数, $\Delta \sigma_{k}$ 为差分元, 例如在一维情形下, $\Delta \sigma_{k}-\Delta x_{i}(k-i)$; 二维情形下, $\Delta \sigma_{k}-\Delta x_{i} \Delta y_{i}(k-(i$, $i))$; 三维情形下, $\Delta o_{k}-\Delta x_{i} \Delta y_{i} \Delta z_{l}(k-(i, j, l))$.

定义范数 $\|F\|^{2}-(F, F)$. 在(2)式中,若将算子 $L$ 进行分解:

$$
L-A+8 \approx \Delta B \text {, }
$$

则(2)式可改写为

$$
\underset{\Delta t}{u^{n+1}-u^{*}}+A u^{*}+8_{n} \Delta t B u^{*}-0,
$$

其中 $B$ 为正定算子, 8 、为待定的耗散系数, $A$ 为与微分算子 $\mathscr{L}$ 相容的空间差分子, 若 $(B u)^{*} \leqslant$ $O(1), 8_{*} \leqslant O(1)$, 则容易证明(5)式与(1)式是相容的.

于是,进一步地可以证明如下定理及其一系列推论.

本文 1989 年 7 月 31 日收到. 1989 年 12 月 30 日收到逄改稿.

- 本研究得到国家七五政关项目的部分资助.

**现为中国科学院大气物理研究所研究生. 
廷理 2 设 $A$ 为反对称算子, $B$ 为正定算子, 且 $(B u)^{n} \leqslant O(1), 2 K_{3} \frac{\Delta t}{\Delta x}<1$, 则当

$$
8_{n}-K_{1} /\left[1-\frac{\Delta t}{\Delta x} K_{2}+\sqrt{\left(1-\frac{\Delta t}{\Delta x} K_{2}\right)^{2}-\left(\frac{\Delta t}{\Delta x} K_{3}\right)^{2}}\right]
$$

时, (5)式为定时间步长的显式完全平方守恒的差分格式, 其中

$$
\left\{\begin{array}{l}
K_{1}-\left\|A u^{n}\right\|^{2} /\left(B u^{*}, u^{*}\right), \\
K_{2}-\left(B u^{*}, A u^{n}\right) \Delta x /\left(B u^{*}, u^{n}\right), \\
K_{3}=\left\|A u^{*}\right\| \cdot\left\|B u^{n}\right\| \cdot \Delta x /\left(B u^{*}, u^{*}\right) .
\end{array}\right.
$$

推论 1 若取 $B u^{n}-\alpha u^{*}+(1-\alpha) u^{n+1}$, 则定理 2 成立. 其中当 $\alpha \leqslant \frac{1}{2}$ 时, 对 $\Delta t$ 没有什么限制; 若 $\alpha>\frac{1}{2}$ 时, 则 $\Delta t$ 必须满足 $\sqrt{(2 \alpha-1)} \Delta t \frac{\left\|A u^{*}\right\|}{\left\|u^{*}\right\|}<1$.

推论 2 若取 $B u^{n}-\delta^{2} u^{n}--\frac{u_{k+1}-2 u_{k}+u_{k-1}}{\rho_{k}(\Delta x)^{2}}$ ( $\rho_{k}$ 为权函数), 则定理 2 成立.

推论 3 若取 $B u^{n}-\frac{A u^{*}-A u^{n-1}}{\Delta t}$, 则定理 2 成立.

推论 4 若取 $B u^{*}-\frac{A \tilde{u}^{n+1}-A u^{*}}{\Delta t}$, 则定理 2 成立, 其中 $\tilde{u}^{n+1}=u^{n}-\Delta t A u^{*}$.

推论 5 若取 $B u^{n}-\frac{A \tilde{u}^{n+1}-A u^{n-1}}{2 \Delta z}$, 则定理 2 成立.

考虑球坐标下的正压初始方程:

$$
\left\{\begin{array}{l}
\frac{\partial u}{\partial t}--\frac{1}{a \cos \theta}\left(\frac{\partial \phi}{\partial \lambda}+u \frac{\partial u}{\partial \lambda}+v^{*} \frac{\partial u}{\partial \theta}\right)+f^{*} v \\
\frac{\partial v}{\partial t}--\frac{1}{a \cos \theta}\left(\cos \theta \frac{\partial \phi}{\partial \theta}+u \frac{\partial v}{\partial \lambda}+v^{*} \frac{\partial v}{\partial \theta}\right)-f^{*} u \\
\frac{\partial \phi}{\partial t}=-\frac{1}{a \cos \theta}\left(\frac{\partial}{\partial \lambda}(u \phi)+\frac{\partial}{\partial \theta}\left(u^{*} \phi\right)\right)
\end{array}\right.
$$

其中 $v^{*}-v \cos \theta, f^{*}-2 \theta \sin \theta+u \operatorname{tg} \theta / a$. 令 $h-\sqrt{\phi}, \bar{U}-h u, \bar{V}-h v$, (8) 式可写为 $\left\{\begin{array}{l}\frac{\partial \bar{U}}{\partial t}--\frac{1}{a \cos \theta}\left\{h \frac{\partial \phi}{\partial \lambda}+\frac{1}{2}\left[\left(\frac{\partial u \bar{U}}{\partial \lambda}+u \frac{\partial \bar{U}}{\partial \lambda}\right)+\left(\frac{\partial v^{*} \bar{U}}{\partial \theta}+v^{*} \frac{\partial \bar{U}}{\partial \theta}\right)\right]\right\}+f^{*} \bar{V}, \\ \frac{\partial \bar{V}}{\partial t}=-\frac{1}{a \cos \theta}\left\{h \cos \theta \frac{\partial \phi}{\partial \theta}+\frac{1}{2}\left[\left(\frac{\partial u \bar{V}}{\partial \lambda}+u \frac{\partial \bar{V}}{\partial \lambda}\right)+\left(\frac{\partial v^{*} \bar{V}}{\partial \theta}+\nu^{*} \frac{\partial \bar{V}}{\partial \theta}\right)\right]\right\}-f^{*} \bar{U}, \\ \frac{\partial \phi}{\partial t}=-\frac{1}{a \cos \theta}\left[\frac{\partial}{\partial \lambda}(u \phi)+\frac{\partial}{\partial \theta}\left(v^{*} \phi\right)\right] .\end{array}\right.$

可以构造相应于(9)式的定步长的显式平方守恒格式:

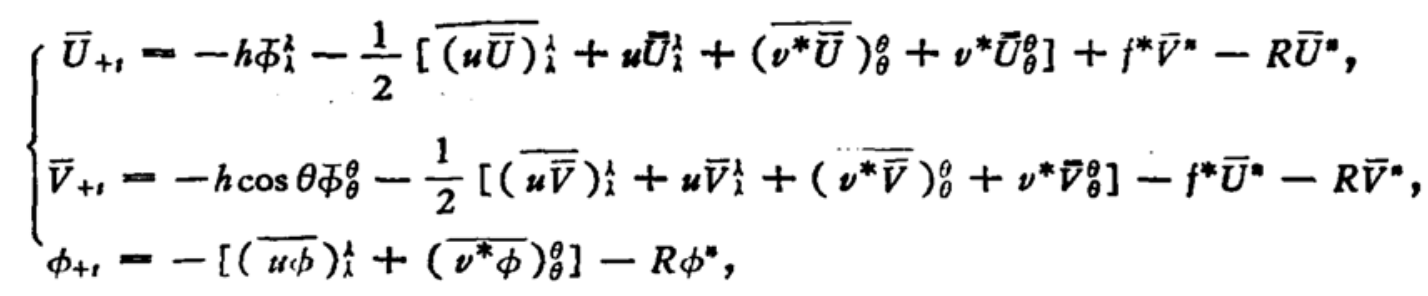

其中 $R F=\varepsilon_{a} \Delta t B \cdot F$. 


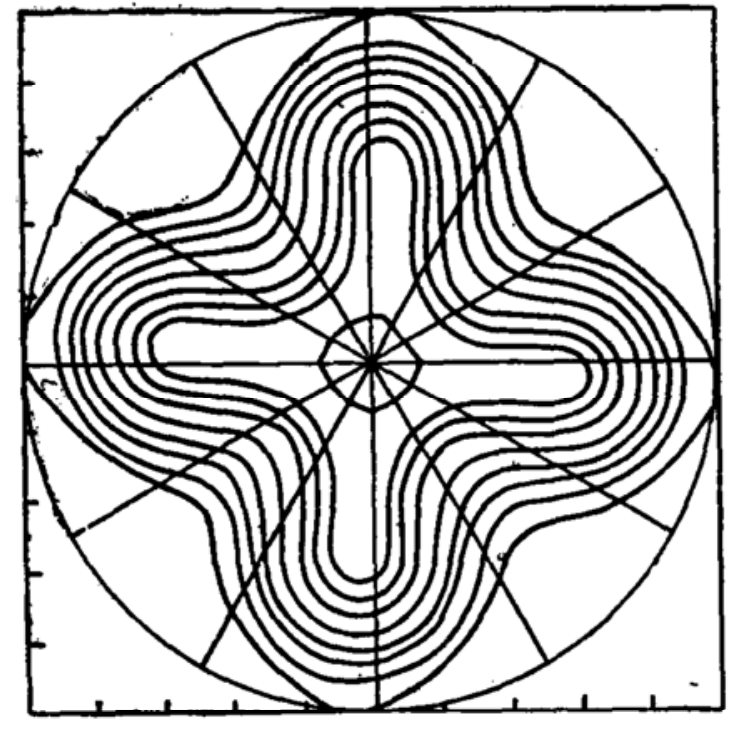

图 1 计算的 R-H 波 (20 天)

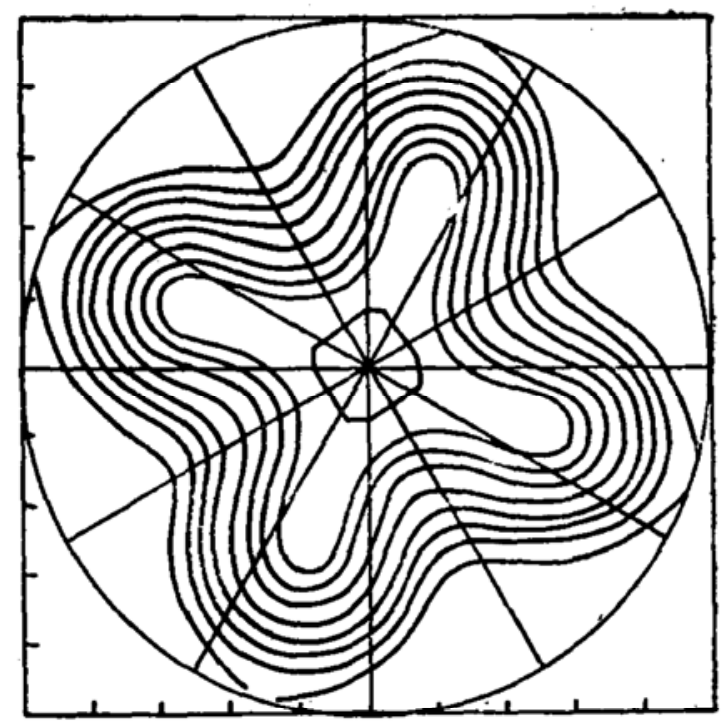

图 2 计算的 $\mathrm{R}-\mathrm{H}$ 波 ( 35 天)

按上述定理 2 的推论 5 选取 $B F^{*}$, 并令 $\Delta \theta-\Delta \lambda-\frac{2 \pi}{80}, \Delta t-300 s$, 用 4 波 RossbyHauritz 波作初始场进行数值积分, 得到较满意的结果. 图 1 和图 2 分别给出积分 20 天和 35 天的计算结果, 可以看到波形基本没有变化,位相移速与真解接近, 大致是每 8 天绕半圈. 表 1 给出总能量 $E^{*}-\sum_{i=1}^{1} \sum_{i=1}^{J} \phi_{i j}^{*} \cos \theta_{i}\left[\left(u_{i j}^{n}\right)^{2}+\left(v_{i j}^{*}\right)^{2}+\phi_{i j}^{*}\right]$ 和总质量 $M^{*}-\sum_{i=1}^{1} \sum_{i=1}^{l} \phi_{i j}^{n} \cos \theta_{i}$ （由于 $\sigma_{i j}-a \Delta \theta \Delta \lambda$ 为常数, 故在原内积的定义中去掉了 $\sigma_{i j}$ ) 随时间演变的数值, 可以看到无 论是总能要还是总质主都非常好地保持了守恒性.

表 1 总能量和总质量的演变

\begin{tabular}{c|c|c}
\hline 天 & 总能量 $E^{*}\left(\mathrm{~m}^{*} / \mathrm{s}^{4}\right)$ & 总质量 $M^{*}\left(\mathrm{~m}^{2} / \mathrm{s}^{2}\right)$ \\
\hline 1 & 18464475564569.4 & 190178908.519517 \\
5 & 18464475564569.3 & 190178908.519517 \\
10 & 18464475564569.5 & 190178908.519517 \\
15 & 18464475564569.4 & 190178908.519517 \\
20 & 18464475564569.4 & 190178908.519517 \\
25 & 18464475564569.5 & 190178908.519517 \\
30 & 18464475564569.6 & 190178908.519517 \\
35 & 18464475564569.9 & 190178908.519517 \\
\hline
\end{tabular}

\section{考文嗝}

[1] 曾庆存、季仲员,つ学学报, 1981, 3: 209--217.

[2] Ji Zhongzhen and Zeng Qingcun, Special Volume of the Journal of the Meteorological Society of Japan, 1987, 255-261.

[ 3] 秦仲贞,中期数值天皆报论文集,气象出版社, 1982, 77-86.

[4] 䇎戻存、张学洪,中国科学, B 辍, 1981, 12: 1355-1366.

[5] 张学洪、曾庆存,大气科学(特刊), 1988, 149-165.

[6]曾戻存、季仲贞、本荣风,大年科学(特刊), 1988, 166-175. 